\title{
Acute monoarthritis: What is the cause of my patient's painful swollen joint?
}

\author{
Lingling Ma MD PhD, Ann Cranney MD MSc, Jayna M. Holroyd-Leduc MD
}

\section{The case}

Early in the evening, a 50-year-old man presents to the emergency department with a 24-hour history of pain and swelling in his right knee. He denies recent injury or trauma. He has no prior history of joint disease or joint surgery and has no family history of arthritis. His medical history includes hypertension, for which he takes hydrochlorothiazide and metoprolol. He drinks 3 beers per day. Given the time of presentation, the results of some laboratory tests, such as Gram staining, may not be available that evening. What should your examination and diagnostic tests focus on to make a diagnosis of this patient's acute monoarthritis?

$\mathrm{A}$ cute monoarthritis is a common clinical presentation in emergency departments and in general medical and rheumatology practices. Arriving at a correct diagnosis is crucial for appropriate treatment. A delay in diagnosis and treatment, particularly with septic arthritis, can result in bacteremia, sepsis, prolonged suffering, joint destruction, disability, a need for prosthetic joint surgery or death. ${ }^{1}$ Therefore, it is important to understand the prevalence of each potential diagnosis and to use an organized and evidencebased clinical approach. Serious errors when managing acute monoarthritis can include failing to perform or delaying joint aspiration and administering antibiotics before aspirating the joint and sending the synovial fluid for culture. Another potential error is starting treatment based on a diagnosis based on laboratory data only (e.g., elevated serum uric acid levels).

In this article we systematically review the evidence for the differential diagnosis of acute monoarthritis and for the diagnostic value of patient history, physical examination and common investigations. We explore specific diagnostic approaches to the most common causes of acute monoarthritis identified in a literature search.

We searched Ovid MEDLINE (1950 to January 2008), EMBASE (1980 to January 2008) and CINAHL (1982 to January 2008) to identify relevant studies. We selected Englishlanguage articles that addressed either the differential diagnosis or the clinical examination of acute monoarthritis, or both, in adults where data were not limited to case reports. Two of us (L.M. and J.M.H-L) independently reviewed and assessed each article for quality and eligibility. Details of the eligibility criteria for inclusion are provided in Appendix 1 (available online at www.cmaj.ca/cgi/content/full/180/1/59/DC1).

Our search yielded 1966 citations. Of these, 58 full-text arti-

\author{
Key points \\ Most common causes of acute monoarthritis: \\ - Gout \\ - Septic arthritis \\ - Rheumatoid arthritis \\ Initial clinical approach for acute monoarthritis: \\ - History and physical examination \\ - Radiography of affected and contralateral joints \\ - Analysis of synovial fluid (gross appearance, crystals, cell \\ count, Gram staining and culture) \\ Important findings in the diagnosis of gout: \\ - Presence of monosodium urate crystals, detected by polarized \\ microscopy \\ - Presence of tophi \\ Important early findings in the diagnosis of septic arthritis: \\ - Leukocyte count in synovial fluid $>50.0 \times 10^{9} / \mathrm{L}$ \\ - $90 \%$ polymorphonuclear cells in the synovial fluid \\ - History of recent joint injury \\ - Presence of overlying skin infection in person with prosthetic \\ hip or knee
}

cles met our inclusion criteria and were retrieved for assessment. A further 38 articles were identified from the reference lists of retrieved articles and from consultation with an expert in the field (details are given in Appendix 2, available online at www.cmaj .ca/cgi/content/full/180/1/59/DC1). We excluded articles that were in languages other than English (9 studies), that lacked relevant data (20 studies), that consisted of reviews that were nonsystematic (34 studies) or of a case report, letter or abstract only (9 studies), that involved children (10 studies) or that involved chronic arthritis (8 studies). We included 6 articles, published between 1980 and 2007, in our final review (Appendix 3, available at www.cmaj.ca/cgi/content/full/180/1/59/DC1). ${ }^{1-6}$

\section{Differential diagnosis of acute monoarthritis}

We identified 4 studies that reported on the differential diagnosis of acute arthritis. ${ }^{2-5}$ The first study described a cohort of 129 patients with acute arthritis who were referred by local

From the Department of Medicine Residency Training Program (Ma), University of Calgary, Calgary, Alta., the Division of Rheumatology (Cranney), Ottawa Health Research Institute, Ottawa, Ont., and the Divisions of Geriatrics and General Internal Medicine and the Knowledge Translation Program (Holroyd-Leduc), University of Calgary, Calgary, Alta. 
general practitioners. The patients were seen over a 12-month period in an acute arthritis clinic in Glasgow, Scotland. ${ }^{4}$ Their arthritis was considered acute if it had been present for 4 weeks or less or if it had involved a flare in 1 joint within the preceding 4 weeks in patients known to have chronic arthritis. The patients were 13-85 years old; 55\% were men. Their diagnostic workups involved a broad number of screening tests, including a routine chest radiograph and other radiology appropriate to the presentation, and laboratory tests for blood urate, erythrocyte sedimentation rate, rheumatoid factor, antinuclear factor, syphilis serology and cultures. Arthrocentesis and analysis of synovial fluid were also performed when indicated and possible.

The second cohort study involved 59 patients assessed for acute monoarthritis in a university-based emergency department in Los Angeles. ${ }^{2}$ Acute arthritis was defined as the presence for less than 2 weeks of any 2 of the following symptoms: tenderness, swelling restricted to the joint and limited joint movement. The patients were $16-75$ years old; $63 \%$ were men. Diagnoses were made with the use of defined criteria that incorporated results from appropriate clinical, radiologic, laboratory and synovial fluid tests. Patients with traumatic arthritis were excluded.

The third cohort study involved 100 consecutive patients experiencing at least 1 swollen and painful joint who underwent

Table 1: Differential diagnosis for acute monoarthritis

\begin{tabular}{|c|c|c|c|c|}
\hline \multirow[b]{2}{*}{ Diagnosis } & \multicolumn{4}{|c|}{ Study; no. (\%) of patients } \\
\hline & $\begin{array}{c}\text { Parker et al. }{ }^{4} \\
n=129\end{array}$ & $\begin{array}{c}\text { Freed et al. }^{2} \\
n=59^{*}\end{array}$ & $\begin{array}{c}\text { Shmerling et al. } .^{5} \\
n=100\end{array}$ & $\begin{array}{c}\text { Jeng et al. }{ }^{3} \\
n=75 t\end{array}$ \\
\hline Gout & $27(21)$ & $9(15)$ & 0 & $20(27)$ \\
\hline $\begin{array}{l}\text { Crystal-induced } \\
\text { arthritis }\end{array}$ & & & $25(25)$ & \\
\hline Septic arthritis & $10(8)$ & $11(19)$ & $8(8)$ & $20(27)$ \\
\hline $\begin{array}{l}\text { Osteoarthritis, } \\
\text { degenerative }\end{array}$ & $6(5)$ & $10(17)$ & $14(14)$ & $11(15)$ \\
\hline Rheumatoid arthritis & $19(15)$ & & $11(11)$ & $12(16)$ \\
\hline Trauma & & & $11(11)$ & \\
\hline $\begin{array}{l}\text { Reactive arthritis, } \\
\text { Reiter syndrome }\end{array}$ & $25(19)$ & 1 (2) & & 7 (9) \\
\hline Lupus & & & & $5(7)$ \\
\hline Miscellaneous & 9 (7) & & $5(5)$ & \\
\hline Psoriatic arthritis & $6(5)$ & & & \\
\hline Soft-tissue problem & 7 (5) & & & \\
\hline Pseudogout & & $2(3)$ & & \\
\hline $\begin{array}{l}\text { Spontaneous } \\
\text { hemarthrosis }\end{array}$ & & $2(3)$ & & \\
\hline Tuberculosis & & $1(2)$ & & \\
\hline Aseptic necrosis & & $1(2)$ & & \\
\hline $\begin{array}{l}\text { Synovial } \\
\text { chondromatosis }\end{array}$ & & $1(2)$ & & \\
\hline Not known & $20(16)$ & $21(36)$ & $26(26)$ & \\
\hline
\end{tabular}

*Patients with traumatic arthritis were excluded.

tOnly patients initially suspected of having septic arthritis were included. diagnostic arthrocentesis. ${ }^{5}$ Diagnoses were based on data collected from electronic health records and from physician notes taken within 48 hours of a joint aspiration. The data collected included patients' histories, findings from physical examinations, radiographs, results of synovial fluid analyses, laboratory results and clinical responses to treatment.

The final study involved 75 patients with suspected septic arthritis who were seen in an emergency department. ${ }^{3}$ Diagnoses were made according to the criteria of the American Rheumatology Association. No further demographic information was available from these last 2 studies.

The cause of the monoarthritis was unknown in a large number of patients $(16 \%-36 \%)$. The most common diagnoses were gout $(15 \%-27 \%)$ and septic arthritis $(8 \%-27 \%)$, followed by osteoarthritis (5\%-17\%) and rheumatoid arthritis $(11 \%-16 \%)$ (Table 1). The variability in causes between studies may be related to differences in the diagnostic criteria they used and the populations they studied.

\section{What should the approach to diagnosing acute monoarthritis be?}

No studies that evaluated patient history or findings from physical examination met our inclusion criteria. However, according to articles published by experts in the field, the initial approach to diagnosing acute monoarthritis should involve the completion of both a history and a physical examination. ${ }^{7.8}$ The history should include a review of symptoms, previous joint disease or trauma, concurrent illnesses, family history of gout, medication use and other risk factors such as travel, sexual history, diet, tick bites, occupational history, alcohol and intravenous drug use. ${ }^{7,8}$ The physical examination should focus on the involved and contralateral joint and surrounding area, along with a general examination to look for other affected joints and for systemic manifestations of disease. ${ }^{7,8}$ By themselves, the patient history and physical examination are usually not enough to make a diagnosis. ${ }^{1,2}$

The most accurate tests for determining the cause of acute monoarthritis were identified by Freed and colleagues ${ }^{2}$ using results from a prospective cohort study involving 59 patients who presented to an emergency department. The tests included radiographs of the involved and con- 
tralateral joints (which led to diagnosis in 8 of 59 cases), examination of the synovial fluid for crystals (7 of 59), Gram staining of the synovial fluid (4 of 59), culture of synovial fluid (4 of 59) and gross appearance of the synovial fluid (2 of 59). In 3 additional patients, a cause was identified based on the early clinical course and the response to treatment (2 cases of probable Neisseria gonorrheae arthritis and 1 case of suspected gout). Of the 4 patients who went on to have a synovial biopsy, the diagnosis was clarified in 3 (tuberculosis in 1 and degenerative dis- ease in 2). The cause in a further 7 patients was identified over the next 1-4 months (degenerative arthritis in 2, septic arthritis in 1, gout or probable gout in 3 and reactive arthritis in 1). Overall, a definitive diagnosis was reached in $64 \%$ of patients. Of the remaining 21 patients, 6 were lost to follow-up, 5 showed no systemic disease or disability, and 1 experienced mild disability. In 9 patients the arthritis resolved spontaneously. These findings suggest that adverse outcomes do not necessarily occur if acute monoarthritis is left undiagnosed after appropriate investigations.

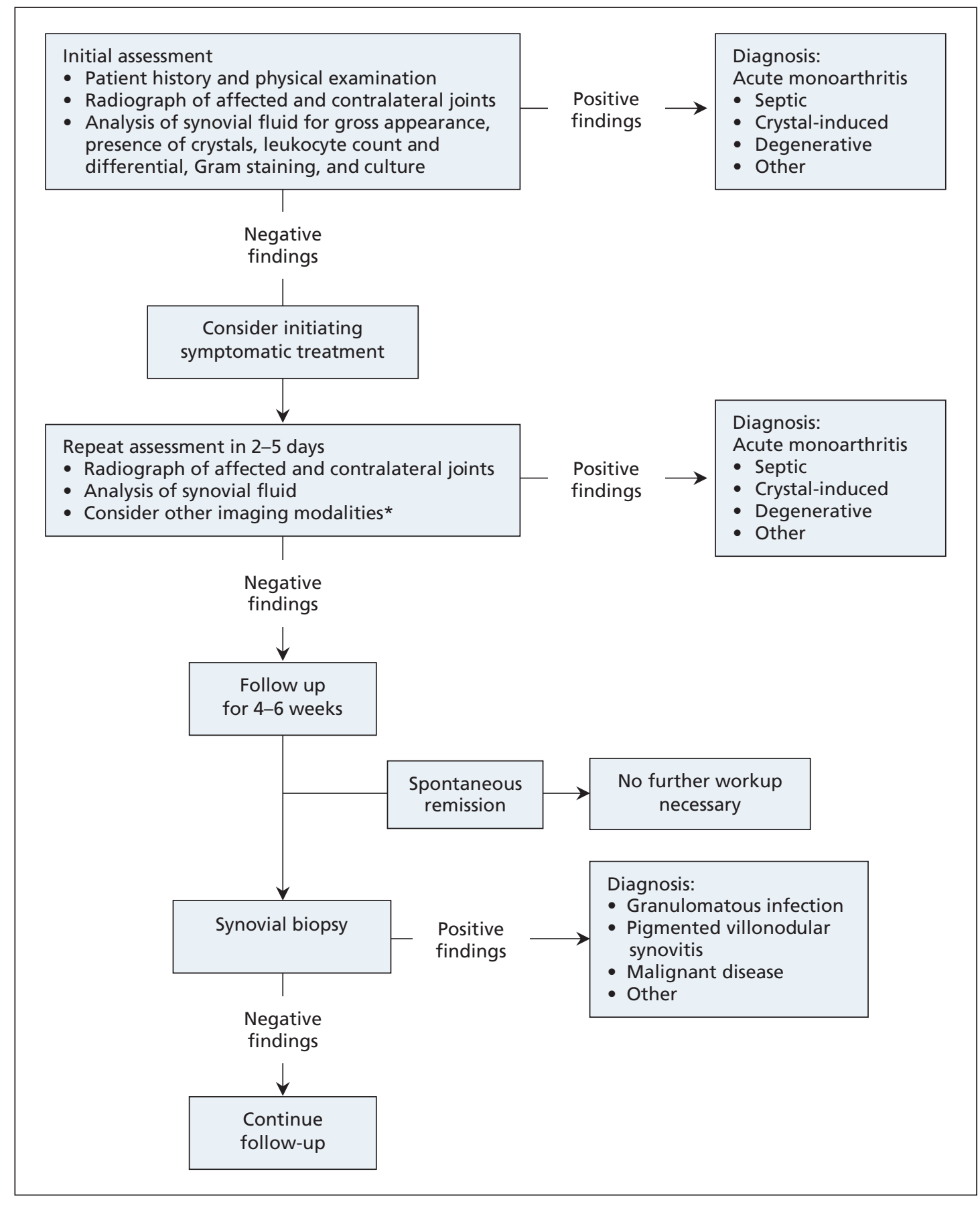

Figure 1: A stepwise approach to the diagnosis of acute monoarthritis. Adapted from Freed et al. ${ }^{2}$ No studies were identified to support this recommendation. 
Based on this study, a diagnostic approach to acute monoarthritis is proposed (Figure 1). ${ }^{2}$ Trauma must be excluded as a cause before this algorithm is used. A key component of this approach is analysis of synovial fluid from the affected joint. Nevertheless, this approach was initially developed over 20 years ago and has not been validated in an independent patient population. Not enough evidence exists to determine whether it should be modified to include the newer diagnostic imaging tools that are now available. It could also be argued that, when a diagnosis is not made after initial investigations, treatment of symptoms should still be considered. Options include acetaminophen $1500 \mathrm{mi} / \mathrm{d}$ or tramadol 150-200 mi/d. Tramadol has a small effect on pain and increased risk of adverse events compared with placebo. ${ }^{9}$ In the absence of contraindications or advanced age, nonsteroidal anti-inflammatory drugs can also be considered to decrease pain and swelling.

An analysis of synovial fluid may be helpful in rapidly distinguishing inflammatory causes of acute monoarthritis (i.e., rheumatoid, crystal-induced and septic arthritis) from noninflammatory causes (i.e., osteoarthritis and trauma). In a prospective cohort study involving 100 patients with acute monoarthritis, Shmerling and colleagues ${ }^{5}$ used an independ-

Table 2: Recommendations of the European League Against Rheumatism for the diagnosis of gout*

Mean score on visual analog scalet $(95 \% \mathrm{Cl})$

In acute attacks, the rapid development of severe pain, swelling and tenderness that reaches its maximum within just 6-12 hours, especially with overlying erythema, is highly suggestive of crystal inflammation, although not specific for gout

For typical presentations of gout, a clinical diagnosis alone is reasonably accurate but not definitive without crystal confirmation

Demonstration of urate crystals in synovial fluid or tophus aspirates permits a definitive diagnosis of gout

A routine search for urate crystals is recommended in all samples of synovial fluid obtained from undiagnosed inflamed joints

Identification of urate crystals in synovial fluid from asymptomatic joints may allow definite diagnosis in intercritical periods

Gout and sepsis may coexist; therefore, when septic arthritis is suspected, Gram staining and culture of synovial fluid should still be performed even if urate crystals are identified

Although the most important risk factor for gout, serum uric acid levels do not confirm or exclude gout: many people with hyperuricemia do not develop gout, and serum levels may be normal during acute attacks

Renal excretion of uric acid should be determined in selected gout patients, especially those with a family history of young onset gout, onset of gout under age 25 or renal calculi

Although radiographs may be useful for the differential diagnosis and may show typical features in chronic gout, they are not useful in confirming the diagnosis of early or acute gout

Risk factors for gout and associated comorbidities should be assessed, including features of metabolic syndrome

Note: $\mathrm{Cl}=$ confidence interval.

*Adapted from Zhang et al. ${ }^{6}$

tThe mean score was derived from individual scores assigned by 20 experts asked to rate the strength of the recommendations on a visual analog scale (from 0-100).

$88(80-96)$
$95(91-98)$

$96(93-100)$

$90(83-97)$

$84(78-91)$

$93(87-99)$

$95(92-99)$

$72(62-81)$

$86(79-94)$

$93(88-98)$ 


\section{How do I diagnose accurately common causes of acute monoarthritis?}

\section{Gout}

One of the most common causes of acute monoarthritis is gout. In 2006, the European League Against Rheumatism developed evidence-based recommendations for the diagnosis of gout. ${ }^{6}$ Its recommendations were based on averaged scores obtained in 13 European countries from 19 participating rheumatologists and 1 expert in evidence-based medicine. They were instructed to base their recommendations on the evidence obtained from a systematic review and on their own clinical expertise.

The systematic review included only studies with a clinical diagnosis of gout. The level of evidence was categorized according to study design. Of the 181 studies reviewed, 83 were diagnostic studies, 86 were management studies, and 12 were both. The validity of the diagnostic tests was evaluated using sensitivity, specificity and LRs. Relative risks or odds ratios were calculated for risk factors and comorbidities. The results were reported as point estimates and 95\% CIs. Although the authors stated that they undertook statistical pooling of data where appropriate, they do not specify the statistical model they used or whether they conducted tests of heterogeneity.

On the basis of the evidence provided and their clinical experience, each of the 20 experts was asked to assign a score for the strength of the recommendations on a visual analog scale (from 0-100). Ten key propositions were generated. An overall score for the strength of each recommendation was calculated as the mean and $95 \%$ CI of the individual scores on the visual analog scale (Table 2).

Based on the systematic review of the European League Against Rheumatism, the presence of tophi (positive LR 40.0, 95\% CI 21.1-75.8) appears to have high clinical diagnostic value. ${ }^{6}$ Risk factors and comorbidities associated with gout include male sex, diabetes, hypertension, obesity, cardiovascular disease, chronic renal failure, use of diuretics, a diet high in purine-rich foods and alcohol consumption (Table 3). ${ }^{6}$ Choi and colleagues ${ }^{11}$ found that, among men with gout, the type of alcohol consumed may be important. Whereas both beer consumption and spirit consumption appear to be associated independently with the risk of gout, wine does not.

Monosodium urate crystals are typically needle-like and show strong negative birefringence. Their identification by polarized microscopy varies according to the skill of the observer but is likely to be positive in an acute gout attack (positive LR 567; 95\% CI 35.5-9053). ${ }^{6}$ The presence of hyperuricemia may also be a useful diagnostic finding when compared with the normal range (i.e., $>$ mean +2 standard deviations) of the local healthy population (positive LR 9.7, 95\% CI 7.5-12.7). Although serum uric acid levels are the most important risk factor for gout, they neither confirm nor exclude it. Many people with hyperuricemia do not have gout; conversely, during acute attacks of gout, serum uric acid levels may be normal. ${ }^{6}$

Several years before the recommendations of the European League Against Rheumatism were developed, the American Rheumatology Association developed diagnostic criteria for acute primary gout. ${ }^{12}$ It compiled data from 706 patients of 38 rheumatology practices across the United States. Patients who had acute primary gout $(n=178)$ were compared with patients who had rheumatoid arthritis $(n=299)$, pseudogout $(n=110)$ or septic arthritis $(n=119)$. From the data collected with the use of a standardized questionnaire, 235 variables were created. Each variable was analyzed in terms of its probability of occurrence in the study groups. Further selection of variables was based on the overall degree of discrimination observed, as well as on clinical judgement. Only the most discriminatory variables were selected. A multivariable analysis was then conducted to verify that the selected variables contributed significantly to the classification of acute gout. From this process, 13 criteria were chosen (Box 1). The presence of 7 or more criteria appears to be required to make a diagnosis of acute gout (sensitivity 74\%; specificity 99\%; positive LR 74; negative LR 0.26). Of note, monosodium urate crystals were found only in aspirates of synovial fluid of patients diagnosed with gout.

Pseudogout can be difficult to distinguish clinically from gout. No articles on pseudogout met our inclusion criteria. However, based on expert opinion, an analysis of synovial fluid for crystals should also include an assessment for calcium pyrophosphate dihydrate crystals, the presence of which helps confirm the diagnosis. ${ }^{13}$ Calcium pyrophosphate dihydrate crystals are polymorphic and often intracellular. Given that they can also be small and show only weak positive birefringence, it is suggested that the detection of crystals be carried out in 2 phases. ${ }^{14}$ Ordinary light microscopy should be used first to

Table 3: Risk factors for gout and septic arthritis

\begin{tabular}{|c|c|c|}
\hline Risk factor & $\begin{array}{c}\text { Gout, }^{6} \\
\text { RR }(95 \% \mathrm{CI})\end{array}$ & $\begin{array}{c}\text { Septic arthritis, }{ }^{1} \\
\text { LR }(95 \% \mathrm{Cl})\end{array}$ \\
\hline \multicolumn{3}{|l|}{ Demographic } \\
\hline Male sex & $7.6(7.5-7.8)$ & - \\
\hline Age $>80$ years & - & $3.5(1.8-7.0)$ \\
\hline \multicolumn{3}{|l|}{ Comorbidities } \\
\hline Diabetes mellitus & $1.1(1.1-1.2)$ & $2.7(1.0-6.9)$ \\
\hline Hypertension & $3.9(1.6-9.7)$ & - \\
\hline Obesity & $3.8(1.2-11.8)$ & - \\
\hline Cardiovascular disease & $1.8(1.7-1.8)$ & - \\
\hline Chronic renal failure & $5.0(4.3-5.7)$ & - \\
\hline Rheumatoid arthritis & - & $2.5(2.0-3.1)$ \\
\hline Recent joint surgery & - & $6.9(3.8-12.0)$ \\
\hline Hip or knee prosthesis & - & $3.1(2.0-4.9)$ \\
\hline Skin infection & - & $2.8(1.7-4.5)$ \\
\hline $\begin{array}{l}\text { Prosthesis with skin } \\
\text { infection }\end{array}$ & - & $15.0(8.1-28.0)$ \\
\hline \multicolumn{3}{|l|}{ Diet and medications } \\
\hline Diuretics & $1.7(1.7-1.8)$ & - \\
\hline Diet high in purine & - & - \\
\hline Meat & $1.4(1.1-1.9)$ & - \\
\hline Seafood & $1.5(1.2-2.0)$ & - \\
\hline $\begin{array}{l}\text { Alcohol (per 10-g daily } \\
\text { increase) }\end{array}$ & $1.2(1.1-1.2)$ & - \\
\hline
\end{tabular}

Note: $\mathrm{Cl}=$ confidence interval, $\mathrm{LR}=$ likelihood ratio, $\mathrm{RR}=$ relative risk 
Box 1: Diagnostic criteria for acute primary gout of the American Rheumatism Association ${ }^{12}$

The presence of 7 or more of the following criteria is required for a diagnosis of acute gout

- More than 1 attack of acute arthritis

- Maximum inflammation developed within 1 day

- Attack of monoarthritis

- Redness observed over joints

- First metatarsophalangeal joint painful and swollen

- Unilateral attack of first metatarsophalangeal joint

- Unilateral attack of tarsal joint

- Tophus (proven or suspected)

- Hyperuricemia

- Asymmetric swelling within a joint on radiograph

- Subcortical cysts without erosions on radiograph

- Monosodium urate monohydrate microcrystals in joint fluid during attack

- Culture of joint fluid negative for organisms during attack

distinguish crystal morphology (e.g., needle-like monosodium urate crystals v. polymorphic calcium pyrophosphate dihydrate crystals). Once crystals have been detected, uncompensated polarized light can be used to distinguish birefringence (e.g., the strong negative birefringence of monosodium urate crystals $\mathrm{v}$. the weakly positive birefringent calcium pyrophosphate dihydrate crystals). When crystals are strongly suspected but are not seen in a sample of synovial fluid, the detection rate may be increased by centrifugation and a repeat search for crystals in the pellet. ${ }^{14}$ However, this step is rarely done in routine clinical practice, according to expert opinion. Rather, a synovial biopsy may help to confirm a diagnosis in this case.

\section{Septic arthritis}

Septic arthritis is another common cause of acute monoarthritis. Significant morbidity can result if treatment is delayed. ${ }^{15}$ Gout and sepsis may coexist. ${ }^{6}$

A recent systematic review evaluated the clinical approach to diagnosing nongonococcal septic arthritis. ${ }^{1}$ The authors used a random-effects model to calculate summary LRs and $95 \%$ CIs to display heterogeneity of results. The 14 studies that were included consisted of case series, case-control series and cohort studies. Since positive culture of synovial fluid has sensitivities of $75 \%-95 \%$, the authors defined the "gold standard" as a positive culture of synovial fluid, a positive Gram stain, a positive blood culture, macroscopic pus aspirated from the joint with no organism isolated, or an appropriate response to antibiotics.

Several risk factors for septic arthritis were identified that are useful when present. They should be part of the initial clinical evaluation of acute monoarthritis (Table 3 ). They include age over 80 years, diabetes mellitus, rheumatoid arthritis, recent joint surgery, hip or knee prosthesis, skin infection, and hip or knee prosthesis with skin infection.

Limited data are available on the symptoms and signs as- sociated with septic arthritis. The specificity and LRs for these could not be calculated because most studies included only patients with septic arthritis. ${ }^{1}$ Joint pain was present in $85 \%$ of patients $(95 \%$ CI $78 \%-90 \%)$, and a history of swelling was present in $78 \%$ (95\% CI 71\%-85\%). Fever (with a sensitivity of $57 \%$ and limited specificity) was not helpful in making a diagnosis.

The most useful laboratory findings in making an early diagnosis of septic arthritis were a leukocyte count in synovial fluid greater than $50.0 \times 10^{9} / \mathrm{L}$ (positive LR 7.7, 95\% CI 5.7-11.0; negative LR $0.42,95 \%$ CI 0.34-0.51), with at least 90\% neutrophils (positive LR 3.4, 95\% CI 2.8-4.2; negative LR $0.34,95 \%$ CI $0.25-0.47) .{ }^{1}$ However, the systematic review was limited by the difficulty associated with defining a gold standard and by a lack of high-quality studies.

A level of tumour necrosis factor- $\alpha$ in synovial fluid greater than $36.2 \mathrm{pg} / \mathrm{mL}$ may also help distinguish septic arthritis from other causes of arthritis. This finding is based on data from a cohort study involving 75 patients who presented to an emergency department with suspected septic arthritis. ${ }^{3}$ Measuring interleukin-1 $\beta$ or interleukin- 6 levels in synovial fluid has no clear diagnostic role. The ability to measure these immune markers may also be limited in clinical practice.

\section{Osteoarthritis}

The knee is commonly affected by osteoarthritis ( $41 \%$ of affected joints). ${ }^{16}$ Although osteoarthritis is typically a chronic condition, acute exacerbations can occur. ${ }^{17,18}$ The American College of Rheumatology (formerly the American Rheumatism Association) has developed 3 sets of diagnostic criteria for osteoarthritis of the knee. ${ }^{17,18}$ The criteria are based on expert consensus using a Delphi procedure and stepwise logistic regression techniques, correlation analysis and classification trees. ${ }^{17}$ The development cohort consisted of 237 patients with knee pain (130 with osteoarthritis; 107 with other diagnoses). Validity testing was performed on an additional 100 consecutive patients with knee pain. The reference standard was a clinical diagnosis of osteoarthritis made by a rheumatologist.

The clinical criteria of the American College of Rheumatology require that the patient have knee pain and at least 3 of the following: age over 50 years; morning stiffness lasting less than 30 minutes duration; crepitus on active range of motion; bony tenderness; bony enlargement; and no palpable warmth (sensitivity 95\%; specificity 69\%; positive LR 3.1; negative LR 0.07). The clinical and radiographic criteria of the American College of Rheumatology require knee pain, osteophytes on radiograph and at least 1 of the following: age over 50 years; crepitus; or morning stiffness lasting less than 30 minutes (sensitivity 91\%; specificity $86 \%$; positive LR 6.5; negative LR 0.10 ). The college's clinical and laboratory criteria require that a patient have knee pain and at least 5 of the following: age over 50 years; morning stiffness lasting less than 30 minutes; crepitus; bony tenderness; bony enlargement; no palpable warmth; an erythrocyte sedimentation rate of less than $40 \mathrm{~mm} / \mathrm{h}$; a rheumatoid factor titre of less than 1:40; and synovial fluid that is clear and viscous with a leukocyte count of less than $2 \times 10^{9} / \mathrm{L}$ (sensitivity $92 \%$; specificity $75 \%$; positive LR 3.7; negative LR 0.11). 


\section{Limitations}

Although we identified several articles that examined the differential diagnosis of acute monoarthritis, in a large number of patients the diagnosis was unknown. Some differences existed between the populations studied. Two studies included patients presenting to an emergency department, ${ }^{2,3} 1$ study included patients presenting to an acute arthritis clinic within 4 weeks of symptom onset, ${ }^{4}$ and 1 study began in a laboratory examining the synovial fluid of hospitalized patients. ${ }^{5}$

The evidence for the diagnostic value of patient history, physical examination and common investigations was limited. We identified only 1 small cohort study that examined an approach to clinical diagnosis and 2 other cohort studies that looked at the role of analysis of synovial fluid. The available literature appears to focus more on diagnosing a specific type of arthritis. Given this limitation, we elected to include data from systematic reviews that focused on common types of arthritis.

No clear gold standards exist for diagnosing the cause of acute monoarthritis. Rather, diagnosis depends on the findings of the clinical examination and supporting investigations and on patient response to treatment. Use of the clinical examination, with or without additional tests, to make a definitive diagnosis is not unique to arthritis. It also occurs in the diagnosis of other conditions such as dementia and Parkinson disease.

Many of the studies we identified in our search were published before 1987. We were unable to find studies that examined the role of newer imaging techniques in the diagnosis of acute monoarthritis.

\section{The case revisited}

Further history from the patient is otherwise unremarkable. Examination of the right knee reveals erythema, tenderness, swelling, an effusion within the knee joint and no palpable bony enlargement. Pain occurs with both active and passive range of motion. There is no crepitus. The left knee is normal on examination. The patient's general physical examination is remarkable only for a body mass index of 30 . No extraarticular findings are noted. A radiograph of both knees reveals a subcortical cyst and swelling within the right knee.

His physician performs an arthrocentesis of the right knee. The synovial fluid appears clear on gross examination, and it is sent for analysis, including leukocyte count, Gram staining and culture. Examination of the synovial fluid under polarizing microscopy reveals bright, negatively birefringent, needle-like intracellular and extracellular crystals that are consistent with monosodium urate crystals. The leukocyte count is $10.0 \times 10^{9} / \mathrm{L}$ with $50 \%$ neutrophils. The Gram stain result will not be available until the following morning and the culture results will not be available for several days.

On the basis of the differential diagnosis of acute monoarthritis (Table 1), the patient's physician assumes that the pretest probability for gout is about $20 \%$ (pretest odds $=0.2 \div[1-0.2]=0.25)$ and that for septic arthritis it is about $15 \%$ (pretest odds $=0.15 \div[1-0.15]=0.18$ ). The presence of monosodium urate crystals in the synovial fluid (positive LR 567) increases the post-test probability for gout to $99 \%$ (post-test odds $=0.25 \times 567=142$ ). Given the leukocyte count in synovial fluid, with less than $90 \%$ neutrophils (negative LR 0.34), the patient's post-test probability for septic arthritis is at most $6 \%$ (post-test odds $=0.18 \times 0.34=0.06$ ).

His physician starts treatment for gout. However, he will follow up the Gram stain and culture results while closely monitoring the patient's clinical response to treatment. If the synovial fluid culture is negative for organisms, the patient will have 7 of the 13 diagnostic criteria proposed by the American Rheumatism Association for acute gout. He does not meet the clinical or the clinical and radiographic diagnostic criteria of the American College of Rheumatology for osteoarthritis of the knee.

This article has been peer reviewed.

Competing interests: None declared.

Contributors: Lingling Ma and Jayna Holroyd-Leduc were responsible for the conception and design of the study. Lingling Ma, Ann Cranney and Jayna Holroyd-Leduc were responsible for the acquisition, analysis and interpretation of data. Jayna Holroyd-Leduc drafted the article. Lingling Ma and Ann Cranney critically revised the article. All of the authors approved the final version for publication.

Acknowledgement: We thank Dr. Scott Richardson, Wright State University, for providing additional references.

Funding: No external funding was received for this research.

\section{REFERENCES}

1. Margaretten ME, Kohlwes J, Moore D, et al. Does this adult patient have septic arthritis? JAMA 2007;297:1478-88.

2. Freed JF, Nies KM, Boyer RS, et al. Acute monoarticular arthritis. A diagnostic approach. JAMA 1980;243:2314-6.

3. Jeng GW, Wang CR, Liu ST, et al. Measurement of synovial tumor necrosis factor-alpha in diagnosing emergency patients with bacterial arthritis. Am J Emerg Med 1997; 15:626-9.

4. Parker JD, Capell HA. An acute arthritis clinic — one year's experience. $\mathrm{Br} \mathrm{J}$ Rheumatol 1986;25:293-5.

5. Shmerling RH, Delbanco TL, Tosteson AN, et al. Synovial fluid tests. What should be ordered? JAMA 1990;264:1009-14.

6. Zhang W, Doherty M, Pascual E, et al. EULAR evidence based recommendations for gout. Part I: Diagnosis. Report of a task force of the Standing Committee for International Clinical Studies Including Therapeutics (ESCISIT). Ann Rheum Dis 2006;65:1301-11.

7. Baker DG, Schumacher HR Jr. Acute monoarthritis. N Engl J Med 1993;329:1013-20.

8. Siva C, Velazquez C, Mody A, et al. Diagnosing acute monoarthritis in adults: practical approach for the family physician. Am Fam Physician 2003;68:83-90.

9. McMahon K, Nelson M, Jones G. Treating the symptoms of osteoarthritis - oral treatments. Aust Fam Physician 2008;37:133-5.

10. Mathews CJ, Kingsley G, Field M, et al. Management of septic arthritis: a systematic review. Ann Rheum Dis 2007;66:440-5.

11. Choi HK, Atkinson K, Karlson EW, et al. Alcohol intake and risk of incident gout in men: a prospective study. Lancet 2004;363:1277-81.

12. Wallace SL, Robinson H, Masi AT, et al. Preliminary criteria for the classification of the acute arthritis of primary gout. Arthritis Rheum 1977;20:895-900.

13. Kohn NN, Hughes RE, McCarty DJ Jr, et al. The significance of calcium phosphate crystals in the synovial fluid of arthritic patients: the "pseudogout syndrome.” II. Identification of crystals. Ann Intern Med 1962;56:738-45.

14. Pascual E, Jovani V. Synovial fluid analysis. Best Pract Res Clin Rheumatol 2005; 19:371-86.

15. Gupta MN, Sturrock RD, Field M. A prospective 2-year study of 75 patients with adult-onset septic arthritis. Rheumatology 2001;40:24-30.

16. Cushnaghan J, Dieppe P. Study of 500 patients with limb joint osteoarthritis. I. Analysis by age, sex, and distribution of symptomatic joint sites. Ann Rheum Dis 1991;50:8-13.

17. Altman R, Asch E, Bloch D, et al. Development of criteria for the classification and reporting of osteoarthritis. Classification of osteoarthritis of the knee. Diagnostic and Therapeutic Criteria Committee of the American Rheumatism Association. Arthritis Rheum 1986;29:1039-49.

18. Jackson JL, O'Malley PG, Kroenke K. Evaluation of acute knee pain in primary care. Ann Intern Med 2003;139:575-88.

Correspondence to: Dr. Jayna M. Holroyd-Leduc, Foothills Medical Centre, 1403-29th St. NW, Calgary AB T2N 2T9; fax 403

944-3699; jayna.holroyd-leduc@albertahealthservices.ca 